PROCEEDINGS OF THE

AMERICAN MATHEMATICAL SOCIETY

Volume 134, Number 6, Pages 1623-1630

S 0002-9939(05)08494-7

Article electronically published on December 15, 2005

\title{
THE RANK OF ELLIPTIC CURVES WITH RATIONAL 2-TORSION POINTS OVER LARGE FIELDS
}

BO-HAE IM

(Communicated by David E. Rohrlich)

\begin{abstract}
Let $K$ be a number field, $\bar{K}$ an algebraic closure of $K, G_{K}$ the absolute Galois group $\operatorname{Gal}(\bar{K} / K), K_{a b}$ the maximal abelian extension of $K$ and $E / K$ an elliptic curve defined over $K$. In this paper, we prove that if all 2-torsion points of $E / K$ are $K$-rational, then for each $\sigma \in G_{K}, E\left(\left(K_{a b}\right)^{\sigma}\right)$ has infinite rank, and hence $E\left(\bar{K}^{\sigma}\right)$ has infinite rank.
\end{abstract}

Let $K$ be a number field, $\bar{K}$ an algebraic closure of $K$, and $G_{K}:=\operatorname{Gal}(\bar{K} / K)$ the absolute Galois group of $\bar{K}$ over $K$. Let $E / K$ be an elliptic curve defined over $K$.

In 4, M. Larsen proved that for any $E / K$ over $K$, there exists a nonempty open subset $\Sigma$ of $G_{K}$ such that for every $\sigma \in \Sigma$, the Mordell-Weil group $E\left(\bar{K}^{\sigma}\right)$ of $E$ over the fixed field under $\sigma$ has infinite rank.

It is natural to ask if such an open subset can be the whole Galois group $G_{K}$. We have a positive answer for elliptic curves defined over $\mathbb{Q}$ and for elliptic curves with certain rational points over the ground field. In 2, we proved that for any elliptic curve $E / \mathbb{Q}$, the rank of $E\left(\overline{\mathbb{Q}}^{\sigma}\right)$ is infinite for every $\sigma \in G_{\mathbb{Q}}$. Our approach in [2] is arithmetic: taking advantage of the modularity of elliptic curves over $\mathbb{Q}$ and the theory of complex multiplication, and constructing an infinite supply of rational points of $E$ consisting of Heegner points. Also, in [1, we proved that for any elliptic curve $E / K$ over a number field $K$ with a $K$-rational point which is neither 2-torsion nor 3-torsion, the rank of $E\left(\bar{K}^{\sigma}\right)$ is infinite for every $\sigma \in G_{K}$. In [1], by a geometric approach, we constructed rational points, essentially by searching for sufficiently rational subvarieties of certain quotients of the $n$-fold product $E^{n}$ of $E$.

This paper has been motivated by [1, 2] and 4]. Extending a method of M. Larsen [4, we prove in this paper that if all 2-torsion points of $E / K$ are $K$ rational, then there are three hyperelliptic curves $X_{1}, X_{2}, X_{3}$ defined over $K$ of genus 1,3 , and 3 respectively forming a biquadratic extension of $\mathbb{P}^{1}$ such that each admits a nonconstant $K$-morphism to $E$. By this result, we prove that if all 2-torsion points of $E / K$ are $K$-rational, then for each $\sigma \in G_{K}, E\left(\left(K_{a b}\right)^{\sigma}\right)$ has infinite rank, where $K_{a b}$ is the maximal abelian extension of $K$, hence $E\left(\bar{K}^{\sigma}\right)$ has infinite rank. Here, we argue by using Diophantine geometry as in [4], which is a completely different method from the one that we used in [2.

Received by the editors January 28, 2005.

2000 Mathematics Subject Classification. Primary 11G05.

(C)2005 American Mathematical Society 
We will need the following lemmas to prove Proposition 4 and we will use the notation in Lemma 2 in the proof of Proposition 4

Lemma 1. If $w_{1}, w_{2}$ and $w_{3}$ are distinct elements in $K$, then there exists an ordered triple $(i, j, k)$ such that $\{i, j, k\}=\{1,2,3\}$ as sets, and $w_{i}+w_{j}-2 w_{k} \neq 0$ and $w_{i}+w_{j}-w_{k} \neq 0$.

Proof. Without loss of generality, suppose $w_{1}+w_{2}-2 w_{3}=0$. Then if $w_{1}+$ $w_{3}-2 w_{2}=0$ or $w_{2}+w_{3}-2 w_{1}=0$, then it implies that $w_{3}=w_{2}$ or $w_{3}=w_{1}$ respectively, which is a contradiction to distinct $w_{i}$ 's. So if $w_{1}+w_{2}-2 w_{3}=0$, then $w_{1}+w_{3}-2 w_{2} \neq 0$ and $w_{2}+w_{3}-2 w_{1} \neq 0$.

If $w_{1}+w_{3}-w_{2} \neq 0$, the ordered triple $(i, j, k)=(1,3,2)$ satisfies the condition. If $w_{1}+w_{3}-w_{2}=0$, then similarly we show that $w_{1}+w_{2}-w_{3} \neq 0$ and $w_{2}+w_{3}-w_{1} \neq 0$. So the ordered triple $(i, j, k)=(2,3,1)$ satisfies the condition.

Lemma 2. For given distinct elements $\alpha, \beta$ and $\gamma \in K$ such that $\beta+\gamma-2 \alpha \neq 0$ and $\beta+\gamma-\alpha \neq 0$, suppose that there exist elements $p, q$, and $s$ in $K$ satisfying the following conditions (11) through (7):

(1) $p, q$ and $s$ are all distinct,

(2) $(p-q)^{3}+(s-q)(p-s)[s(\alpha-\beta)+p(\gamma-\alpha)+q(\beta-\gamma)] \neq 0$,

(3) $(p-q)^{3}(\beta+\gamma-\alpha)+\alpha(s-q)(p-s)[s(\alpha-\beta)+p(\gamma-\alpha)+q(\beta-\gamma)] \neq 0$,

(4) $(p-q)^{3} \alpha+(\beta+\gamma-\alpha)(s-q)(p-s)[s(\alpha-\beta)+p(\gamma-\alpha)+q(\beta-\gamma)] \neq 0$,

(5) $(p-q)^{3}(\gamma-\alpha)+(\alpha-\beta)(s-q)(p-s)[s(\alpha-\beta)+p(\gamma-\alpha)+q(\beta-\gamma)] \neq 0$,

(6) $(p-q)^{3}(\beta-\alpha)+(\alpha-\gamma)(s-q)(p-s)[s(\alpha-\beta)+p(\gamma-\alpha)+q(\beta-\gamma)] \neq 0$,

(7) $s(\alpha-\beta)+p(\gamma-\alpha)+q(\beta-\gamma) \neq 0$.

Then let $F$ and $G$ be the left-hand sides of (2) and (3) respectively, and put

$$
\begin{aligned}
& t=\frac{G}{F}, \\
& A=(\beta+\gamma-2 \alpha)(s(\alpha-\beta)+p(\gamma-\alpha)+q(\beta-\gamma)), \\
& B_{1}=(t-\alpha)(s(\alpha-\beta)+p(\gamma-\alpha)+q(\beta-\gamma))(p+q), \\
& B_{2}=(t-(\beta+\gamma-\alpha))\left(s^{2}(\alpha-\beta)+s(p-q)(2 \gamma-\alpha-\beta)+p q(\beta-\alpha)\right), \\
& C_{1}=(t-\alpha)(s(\alpha-\beta)+p(\gamma-\alpha)+q(\beta-\gamma)) p q, \\
& C_{2}=(t-(\beta+\gamma-\alpha))\left(s^{2}(p(\gamma-\beta)+q(\alpha-\gamma))+s p q(\beta-\alpha)\right) .
\end{aligned}
$$

Define three polynomials in $x$ :

$h_{1}(x)=A(t-\beta) x^{2}-\left((\gamma-\alpha) B_{1}-(\alpha-\beta) B_{2}\right) x+\left((\gamma-\alpha) C_{1}-(\alpha-\beta) C_{2}\right)$,

$h_{2}(x)=A(t-\gamma) x^{2}-\left((\beta-\alpha) B_{1}-(\alpha-\gamma) B_{2}\right) x+\left((\beta-\alpha) C_{1}-(\alpha-\gamma) C_{2}\right)$,

$h_{3}(x)=A x^{2}-\left(B_{1}-B_{2}\right) x+\left(C_{1}-C_{2}\right)$.

Then, $A \neq 0$ and $t \neq 0, \beta+\gamma, \beta, \gamma, \beta+\gamma-\alpha, \alpha$. In particular, the $h_{i}$ are quadratic polynomials in $x$ defined over $K$.

Proof. Conditions (3) through (6) imply that $t \neq 0, \beta+\gamma, \beta, \gamma$. Since $\beta+\gamma-2 \alpha \neq 0$, conditions (11) and (7) imply that $t \neq \beta+\gamma-\alpha$ and $t \neq \alpha$. By condition (7) and the assumption that $\beta+\gamma-2 \alpha \neq 0$, we see that $A \neq 0$.

Since $A \neq 0$ and $t \neq \beta, \gamma$ and $t, A, B_{1}, B_{2}, C_{1}, C_{2}$ are in $K$, the $h_{i}$ are quadratic polynomials in $x$ defined over $K$.

Lemma 3. For given distinct elements $\alpha, \beta$ and $\gamma \in K$ such that $\beta+\gamma-2 \alpha \neq 0$ and $\beta+\gamma-\alpha \neq 0$, there exist elements $p, q$, and $s \in K$ satisfying the following 
conditions:

$p, q$, and s satisfy conditions (1) through (7) in Lemma 2,

$p, q$ and $s$ are not equal to $\frac{s(p(\gamma-\beta)+q(\alpha-\gamma))+p q(\beta-\alpha)}{s(\alpha-\beta)+p(\gamma-\alpha)+q(\beta-\gamma)}$,

$\alpha(p-s)-\beta(q-s) \neq 0$,

each quadratic polynomial $h_{i}(x)$ defined in Lemma 2 has no double zero,

any two of three quadratic polynomials $h_{i}(X)$ have no common zero,

$p, q$, and $s$ are not zeros of any of $h_{i}(x)$.

Proof. Note that the set of triples $(p, q, s)$ satisfying each condition from (1) through (9) is a finite number of intersections of nonempty open subsets in $\mathbb{A}^{3}(K)$ defined by the nontrivial polynomials over $K$ given in each condition. So by the Hilbert irreducibility theorem [3. Chapter 9$]$, the set of $(p, q, s) \in \mathbb{A}^{3}(K)$ satisfying conditions (11) through (9) is infinite.

For any $p, q, s$ satisfying (11) through (9), Lemma 2 shows that $h_{i}(x)$ are quadratic polynomials. Let $D\left(h_{i}\right)$ denote the discriminant of the quadratic polynomial $h_{i}$. Then,

$$
\begin{aligned}
& D\left(h_{1}\right)=\left((\gamma-\alpha) B_{1}-(\alpha-\beta) B_{2}\right)^{2}-4 A(t-\beta)\left((\gamma-\alpha) C_{1}-(\alpha-\beta) C_{2}\right), \\
& D\left(h_{2}\right)=\left((\beta-\alpha) B_{1}-(\alpha-\gamma) B_{2}\right)^{2}-4 A(t-\gamma)\left((\beta-\alpha) C_{1}-(\alpha-\gamma) C_{2}\right), \\
& D\left(h_{3}\right)=\left(B_{1}-B_{2}\right)^{2}-4 A\left(C_{1}-C_{2}\right) .
\end{aligned}
$$

We can show that the discriminants $D\left(h_{i}\right)$ are nontrivial rational functions in $p, q$ and $s$. For example, we show 1 that the coefficients of the $s^{10}$-term in $F^{2} D\left(h_{1}\right)$ (resp. $\left.F^{2} D\left(h_{2}\right), F^{2} D\left(h_{3}\right)\right)$ is $-(\alpha-\beta)^{6}(\beta+\gamma-2 \alpha)^{2}\left(\right.$ resp. $-(\alpha-\beta)^{4}(\alpha-\gamma)^{2}(\beta+\gamma-2 \alpha)^{2}$, $\left.-(\alpha-\beta)^{4}(\beta+\gamma-2 \alpha)^{2}\right)$, which is not zero by the assumption on $\alpha, \beta$ and $\gamma$. Also the $h_{i}$ have the nontrivial relation $h_{1}-h_{2}=(\gamma-\beta) h_{3}$. The set of triples $(p, q, s)$ at which $D\left(h_{i}\right)$ for some $i=1,2,3$ vanishes or two of $h_{1}(x), h_{2}(x), h_{3}(x)$ have a common zero is a finite number of unions of proper Zariski closed sets in $\mathbb{A}^{3}(K)$. Its complementary set is a finite number of intersections of nonempty Zariski open sets, which is infinite by the Hilbert irreducibility theorem. Also, the set of triples $(p, q, s)$ such that $p, q$, and $s$ are not zeros of any $h_{i}(x)$ is also an intersection of three nonempty Zariski open sets, which is infinite. So the set of $(p, q, s) \in \mathbb{A}^{3}(K)$ satisfying conditions (11) through (12) is a finite number of intersections of nonempty Zariski open sets, hence it is nonempty (in fact, it is infinite).

Proposition 4. If all 2-torsion points of $E / K$ are $K$-rational (i.e. $E[2] \subset E(K)$ ), then there exist three hyperelliptic curves $X_{1}, X_{2}, X_{3}$ defined over $K$ of genus $1,3,3$ respectively which map onto $E$ over $K$ and are defined by

$$
\left\{\begin{array}{l}
X_{1}: y^{2}=a f(x) g(x), \\
X_{2}: y^{2}=b f(x) h(x), \\
X_{3}: y^{2}=\operatorname{abg}(x) h(x)
\end{array}\right.
$$

for some constants $a, b \in K$, and some polynomials $f, g, h \in K[x]$. Hence the curves $X_{1}, X_{2}$ and $X_{3}$ form a biquadratic extension of $\mathbb{P}^{1}$ over $K$.

\footnotetext{
${ }^{1}$ This can be shown by a moderate amount of direct calculation and has been verified using Maple (full documentation available upon request).
} 
Proof. Let $y^{2}=(x-\alpha)(x-\beta)(x-\gamma)$ be a Weierstrass equation of $E$. Here, $\alpha, \beta$, and $\gamma$ are distinct elements in $K$, since the 2-torsion points of $E$ are $K$-rational. Then, by Lemma 1 we may assume that

$$
\beta+\gamma-2 \alpha \neq 0 \text { and } \beta+\gamma-\alpha \neq 0 .
$$

The main strategy for proving the proposition is to construct three curves defined by

$X_{1}: y^{2}=a(x-p)(x-q)(x-s)(x-r)$,

$X_{2}: y^{2}=b(x-p)(x-q)\left(x-x_{1}\right)\left(x-x_{2}\right)\left(x-x_{3}\right)\left(x-y_{1}\right)\left(y-y_{2}\right)\left(y-y_{3}\right)$,

$X_{3}: y^{2}=a b(x-s)(x-r)\left(x-x_{1}\right)\left(x-x_{2}\right)\left(x-x_{3}\right)\left(x-y_{1}\right)\left(y-y_{2}\right)\left(y-y_{3}\right)$

together with three dominant morphisms $\phi_{i}: X_{i} \rightarrow E$ for $i=1,2,3$ defined by $K$-rational functions which map the set of ramification points of each $X_{i}$ over $\mathbb{P}^{1}$ onto the set of ramification points of $E$ over $\mathbb{P}^{1}$ as follows:

$$
\begin{aligned}
\phi_{1} & :(p, 0) \mapsto(\alpha, 0), \quad(q, 0) \mapsto(\beta, 0), \quad(r, 0) \mapsto(\gamma, 0), \text { and }(s, 0) \mapsto \infty, \\
\phi_{2}: & (p, 0),(q, 0) \mapsto(\alpha, 0), \quad\left(x_{1}, 0\right),\left(x_{2}, 0\right) \mapsto(\beta, 0), \\
& \left(y_{1}, 0\right),\left(y_{2}, 0\right) \mapsto(\gamma, 0), \text { and }\left(x_{3}, 0\right),\left(y_{3}, 0\right) \mapsto \infty, \\
\phi_{3}: & (r, 0),(s, 0) \mapsto(\alpha, 0), \quad\left(x_{1}, 0\right),\left(x_{2}, 0\right) \mapsto(\gamma, 0), \\
& \left(y_{1}, 0\right),\left(y_{2}, 0\right) \mapsto(\beta, 0), \text { and }\left(x_{3}, 0\right),\left(y_{3}, 0\right) \mapsto \infty .
\end{aligned}
$$

First, under the assumption (13), by Lemma 3, we can choose $p, q, s \in K$ satisfying conditions (11) through (12).

Then we find $X_{1}$ and a dominant morphism $\phi_{1}: X_{1} \rightarrow E$ satisfying (14) in terms of the given $p, q, s, \alpha, \beta$ and $\gamma$. Since $X_{1}$ has genus 1 and $\phi_{1}$ sends $(s, 0) \in X_{1}$ to $\infty \in E$ as a dominant map, we try to set $\phi_{1}(x, y)=\left(\frac{c_{1} x+c_{0}}{(x-s)}, \frac{c_{3}}{(x-s)^{2}} y\right)$ as rational functions for some constants $c_{i}$ and try to find constants $r$ and $c_{i}$ in terms of $p, q, s, \alpha, \beta$ and $\gamma$. For $\phi_{1}$ to satisfy condition (14), we need three relations,

$$
c_{1} p+c_{0}=\alpha(p-s), \quad c_{1} q+c_{0}=\beta(q-s), \quad c_{1} r+c_{0}=\gamma(r-s) .
$$

Also, for $\phi_{1}$ to be a dominant morphism to $E$, the following must be valid:

$$
Z\left(\phi_{1}(x, y)\right)=0, \text { where } Z(x, y)=y^{2}-(x-\alpha)(x-\beta)(x-\gamma) .
$$

Next, we find $X_{2}$ and $X_{3}$ as well as dominant morphisms $\phi_{2}$ and $\phi_{3}$ satisfying conditions (15) and (16). Since $\left(x_{3}, 0\right)$ and $\left(y_{3}, 0\right) \in X_{i}$ are mapped to $\infty$ under $\phi_{i}$ for $i=2,3$ and two distinct points $\left(x_{1}, 0\right)$ and $\left(x_{2}, 0\right)$ (and $\left(y_{1}, 0\right)$ and $\left(y_{2}, 0\right)$ ) are mapped onto the same point in $E, \phi_{i}$ needs to be defined by rational functions with polynomials of degree at least 2 as numerators and with $\left(x-x_{3}\right)\left(x-y_{3}\right)$ as denominators. We let for some constants $d_{i}$ and $e_{i}$,

$$
\begin{gathered}
\phi_{2}(x, y)=\left(\frac{d_{2} x^{2}+d_{1} x+d_{0}}{\left(x-x_{3}\right)\left(x-y_{3}\right)}, \frac{d_{3}}{\left(\left(x-x_{3}\right)\left(x-y_{3}\right)\right)^{2}} y\right), \text { and } \\
\phi_{3}(x, y)=\left(\frac{e_{2} x^{2}+e_{1} x+e_{0}}{\left(x-x_{3}\right)\left(x-y_{3}\right)}, \frac{e_{3}}{\left(\left(x-x_{3}\right)\left(x-y_{3}\right)\right)^{2}} y\right) .
\end{gathered}
$$


In this setting, for $\phi_{2}$ and $\phi_{3}$ to satisfy conditions (15) and (16) respectively, the following six equations must hold:

$(* *)$

$$
\left\{\begin{array}{l}
\left(d_{2}-\alpha\right) x^{2}+\left(d_{1}+\alpha\left(x_{3}+y_{3}\right)\right) x+d_{0}-\alpha x_{3} y_{3}=\left(d_{2}-\alpha\right)(x-p)(x-q), \\
\left(d_{2}-\beta\right) x^{2}+\left(d_{1}+\beta\left(x_{3}+y_{3}\right)\right) x+d_{0}-\beta x_{3} y_{3}=\left(d_{2}-\beta\right)\left(x-x_{1}\right)\left(x-x_{2}\right), \\
\left(d_{2}-\gamma\right) x^{2}+\left(d_{1}+\gamma\left(x_{3}+y_{3}\right)\right) x+d_{0}-\gamma x_{3} y_{3}=\left(d_{2}-\gamma\right)\left(x-y_{1}\right)\left(x-y_{2}\right), \\
\left(e_{2}-\alpha\right) x^{2}+\left(e_{1}+\alpha\left(x_{3}+y_{3}\right)\right) x+e_{0}-\alpha x_{3} y_{3}=\left(e_{2}-\alpha\right)(x-r)(x-s), \\
\left(e_{2}-\beta\right) x^{2}+\left(e_{1}+\beta\left(x_{3}+y_{3}\right)\right) x+e_{0}-\beta x_{3} y_{3}=\left(e_{2}-\beta\right)\left(x-y_{1}\right)\left(x-y_{2}\right), \\
\left(e_{2}-\gamma\right) x^{2}+\left(e_{1}+\gamma\left(x_{3}+y_{3}\right)\right) x+e_{0}-\gamma x_{3} y_{3}=\left(e_{2}-\gamma\right)\left(x-x_{1}\right)\left(x-x_{2}\right) .
\end{array}\right.
$$

Then we find constants $d_{j}, e_{j}, x_{j}$, and $y_{j}$ by equating the coefficients of constant terms, $x$ and $x^{2}$-terms in each equation in $(* *)$ and requiring that

$$
Z\left(\phi_{i}(x, y)\right)=0 \text {, where } Z(x, y)=y^{2}-(x-\alpha)(x-\beta)(x-\gamma) \text { and } i=2,3 .
$$

Now we solve for $r, x_{i}, y_{i}, c_{i}, d_{i}, e_{i}$ and give a solution to all equations from (17) to $(* *)$ and (19) listed in the above for $X_{i}$ and $\phi_{i}$ as follows.

A moderate amount of direct calculation from (17) gives the following in terms of $p, q, s, \alpha, \beta$, and $\gamma$ :

$$
\begin{aligned}
& r=\frac{s(p(\gamma-\beta)+q(\alpha-\gamma))+p q(\beta-\alpha)}{s(\alpha-\beta)+p(\gamma-\alpha)+q(\beta-\gamma)} \in K, \\
& c_{0}=\frac{(\beta-\alpha) p q+s(\alpha q-\beta p)}{(p-q)} \in K, \\
& c_{1}=\frac{\alpha(p-s)-\beta(q-s)}{(p-q)} \in K \backslash\{0\} .
\end{aligned}
$$

Conditions (11), (7) and (8) imply that $r, c_{0}$ and $c_{1}$ are well-defined and that $p, q, s$ and $r$ are all distinct. Also, $c_{1}$ is nonzero by condition (9).

If we let

$$
\begin{aligned}
& c_{3}=(\alpha-\beta) \in K \backslash\{0\}, \\
& a=\frac{(s-q)(p-s)[s(\alpha-\beta)+p(\gamma-\alpha)+q(\beta-\gamma)]}{(p-q)^{3}} \in K \backslash\{0\},
\end{aligned}
$$

then $a \neq 0$ by conditions (11) and (7) and $\phi_{1}$ is a nonconstant map since $c_{3} \neq 0$. We can verify 2 that $\phi_{1}$ is a dominant morphism over $K$ satisfying condition (14) and that (18) holds.

Next we let

$$
b=(t-\alpha)(t-\beta)(t-\gamma) \in K \text {. (Then } b \neq 0 \text { by Lemma 2) }
$$

We can show ${ }^{2}$ that $a$ defined in (24) equals $\frac{\beta+\gamma-\alpha-t}{t-\alpha}$. Hence we get

$$
a b=(\beta-t)(\gamma-t)(\beta+\gamma-\alpha-t) \in K \backslash\{0\} .
$$

Then, for the quadratic polynomials $h_{i}(x)$ defined in Lemma 2, let $x_{1}$ and $x_{2}$ be zeros of $h_{1}(x), y_{1}$ and $y_{2}$ zeros of $h_{2}(x)$, and $x_{3}$ and $y_{3}$ zeros of $h_{3}(x)$. Then $p, q, s, r, x_{i}, y_{j}$ are all distinct by conditions (11), (10), (11) and (12).

\footnotetext{
${ }^{2}$ This can be shown by a moderate amount of direct calculation and has been verified using Maple (full documentation available upon request).
} 
Using the same notation as in Lemma 2 we define the curves $X_{i}$ and the maps $\phi_{i}: X_{i} \rightarrow E$ for $i=1,2,3$ as follows (note that $X_{1}$ and $\phi_{1}$ have been found in (20) through (24)):

$$
\begin{aligned}
X_{1}: y^{2} & =a(x-p)(x-q)(x-s)\left(x-\frac{s(p(\gamma-\beta)+q(\alpha-\gamma))+p q(\beta-\alpha)}{s(\alpha-\beta)+p(\gamma-\alpha)+q(\beta-\gamma)}\right), \\
X_{2}: y^{2} & =\frac{(t-\alpha)}{A^{3}}(x-p)(x-q) h_{1}(x) h_{2}(x) h_{3}(x) \\
( & \left.=b(x-p)(x-q)\left(x-x_{1}\right)\left(x-x_{2}\right)\left(x-y_{1}\right)\left(x-y_{2}\right)\left(x-x_{3}\right)\left(x-y_{3}\right)\right), \\
X_{3}: y^{2} & =\frac{(\beta+\gamma-\alpha-t)}{A^{3}}(x-s)\left(x-\frac{s(p(\gamma-\beta)+q(\alpha-\gamma))+p q(\beta-\alpha)}{s(\alpha-\beta)+p(\gamma-\alpha)+q(\beta-\gamma)}\right) h_{1}(x) h_{2}(x) h_{3}(x) \\
( & \left.=a b(x-s)(x-r)\left(x-x_{1}\right)\left(x-x_{2}\right)\left(x-y_{1}\right)\left(x-y_{2}\right)\left(x-x_{3}\right)\left(x-y_{3}\right)\right), \\
\phi_{1}(x, y) & =\left(\frac{(\alpha(p-s)-\beta(q-s)) x+(\beta-\alpha) p q+s(\alpha q-\beta p)}{(p-q)(x-s)}, \quad \frac{(\alpha-\beta)}{(x-s)^{2}} y\right), \\
\phi_{2}(x, y) & =\left(\frac{h_{1}(x)+\beta h_{3}(x)}{h_{3}(x)}, \quad \frac{A^{2}}{h_{3}(x)^{2}} y\right) \\
( & =\left(\frac{A t x^{2}-\left((\beta+\gamma-\alpha) B_{1}-\alpha B_{2}\right) x+\left((\beta+\gamma-\alpha) C_{1}-\alpha C_{2}\right)}{A x^{2}-\left(B_{1}-B_{2}\right) x+\left(C_{1}-C_{2}\right)},\right. \\
\phi_{3}(x, y) & \left.\left.=\left(\frac{-h_{2}(x)+\beta h_{3}(x)}{h_{3}(x)}, \quad \frac{A^{2}}{h_{3}(x)^{2}} y\right) \quad \frac{A^{2}}{\left(A x^{2}-\left(B_{1}-B_{2}\right) x+\left(B_{1}\right) x+\left(C_{1}-C_{2}\right)\right)^{2}} y\right)\right), \\
( & \left.=\left(\frac{A(\beta+\gamma-t) x^{2}-\left(\alpha B_{1}-(\beta+\gamma-\alpha) B_{2}\right) x+\left(\alpha C_{1}-(\beta+\gamma-\alpha) C_{2}\right)}{A x^{2}-\left(B_{1}-B_{2}\right) x+\left(C_{1}-C_{2}\right)} y\right)\right) .
\end{aligned}
$$

Then, since $p, q, s, r, x_{i}, y_{i}$ are all distinct and $a \neq 0, b \neq 0, A \neq 0, t \neq 0, \alpha, \beta+$ $\gamma-\alpha, \beta+\gamma, X_{1}, X_{2}, X_{3}$ are nonsingular curves of genus 1,3,3 respectively and $\phi_{i}$ are nonconstant maps. And since $A, a, b \in K$ and $h_{i}(x)$ are defined over $K, X_{i}$ and $\phi_{i}$ are defined over $K$.

Before we show that the maps $\phi_{2}$ and $\phi_{3}$ defined above satisfy (15) and (16) respectively (i.e. satisfy the equations in $(* *)$ ) and satisfy (19), we verify the following identities:

$$
\begin{aligned}
h_{1}(x)+(\beta-\alpha) h_{3}(x) & =A(t-\alpha)(x-p)(x-q), \\
h_{1}(x)+(\beta-\gamma) h_{3}(x) & =h_{2}(x), \\
-h_{2}(x)+(\beta-\alpha) h_{3}(x) & =A(\beta+\gamma-\alpha-t)(x-s)(x-r), \\
-h_{2}(x)+(\beta-\gamma) h_{3}(x) & =-h_{1}(x) .
\end{aligned}
$$

For the validity of (15) for $\phi_{2}$, we show that

$\phi_{2}(p, 0)=\left(\frac{h_{1}(p)+\beta h_{3}(p)}{h_{3}(p)}, 0\right)=\left(\frac{(\alpha-\beta) h_{3}(p)+\beta h_{3}(p)}{h_{3}(p)}, 0\right)=(\alpha, 0)$ by (25),

$\phi_{2}(q, 0)=\left(\frac{h_{1}(q)+\beta h_{3}(q)}{h_{3}(q)}, 0\right)=\left(\frac{(\alpha-\beta) h_{3}(q)+\beta h_{3}(q)}{h_{3}(p)}, 0\right)=(\alpha, 0)$ by (25) $)$

for $i=1,2, \phi_{2}\left(x_{i}, 0\right)=\left(\frac{h_{1}\left(x_{i}\right)+\beta h_{3}\left(x_{i}\right)}{h_{3}\left(x_{i}\right)}, 0\right)=(\beta, 0)$, since $h_{1}\left(x_{i}\right)=0$,

${ }^{3}$ This can be shown by a moderate amount of direct calculation and has been verified using Maple (full documentation available upon request). 
$\phi_{2}\left(y_{i}, 0\right)=\left(\frac{h_{1}\left(y_{i}\right)+\beta h_{3}\left(y_{i}\right)}{h_{3}\left(y_{i}\right)}, 0\right)=\left(\frac{(\gamma-\beta) h_{3}\left(y_{i}\right)+\beta h_{3}\left(y_{i}\right)}{h_{3}\left(y_{i}\right)}, 0\right)=(\gamma, 0)$ by (26), since $h_{2}\left(y_{i}\right)=0$, and $\phi_{2}\left(x_{3}, 0\right)=\infty=\phi_{2}\left(x_{3}, 0\right)$, since $h_{3}\left(x_{3}\right)=0=h_{3}\left(y_{3}\right)$.

Similarly, (16) can be verified for $\phi_{3}$.

For the validity of (19) for $i=2$, by using (25) and (26), we show that

$$
\begin{aligned}
\frac{A^{4}}{h_{3}(x)^{4}} y^{2} & =\left(\frac{h_{1}(x)+\beta h_{3}(x)}{h_{3}(x)}-\alpha\right)\left(\frac{h_{1}(x)+\beta h_{3}(x)}{h_{3}(x)}-\beta\right)\left(\frac{h_{1}(x)+\beta h_{3}(x)}{h_{3}(x)}-\gamma\right) \\
& =\frac{\left(h_{1}(x)+(\beta-\alpha) h_{3}(x)\right)\left(h_{1}(x)\right)\left(h_{1}(x)+(\beta-\gamma) h_{3}(x)\right)}{h_{3}(x)^{3}} \\
& =\frac{A(t-\alpha)(x-p)(x-q) h_{1}(x) h_{2}(x)}{h_{3}(x)^{3}}
\end{aligned}
$$

which reduces to $y^{2}=\frac{(t-\alpha)}{A^{3}}(x-p)(x-q) h_{1}(x) h_{2}(x) h_{3}(x)$ and defines $X_{2}$.

Similarly, for the validity of (19) for $i=3$, by using (27) and (28), we show that

$$
\begin{aligned}
& \frac{A^{4}}{h_{3}(x)^{4}} y^{2} \\
& \quad=\left(\frac{-h_{2}(x)+\beta h_{3}(x)}{h_{3}(x)}-\alpha\right)\left(\frac{-h_{2}(x)+\beta h_{3}(x)}{h_{3}(x)}-\beta\right)\left(\frac{-h_{2}(x)+\beta h_{3}(x)}{h_{3}(x)}-\gamma\right) \\
& \quad=\frac{\left(-h_{2}(x)+(\beta-\alpha) h_{3}(x)\right)\left(-h_{2}(x)\right)\left(-h_{2}(x)+(\beta-\gamma) h_{3}(x)\right)}{h_{3}(x)^{3}} \\
& \quad=\frac{A(\beta+\gamma-\alpha-t)(x-s)(x-r)\left(-h_{2}(x)\right)\left(-h_{1}(x)\right)}{h_{3}(x)^{3}}
\end{aligned}
$$

which reduces to $y^{2}=\frac{(\beta+\gamma-\alpha-t)}{A^{3}}(x-s)(x-r) h_{1}(x) h_{2}(x) h_{3}(x)$ and defines $X_{3}$.

Note that all $X_{i}$ and $\phi_{i}$ are defined over $K$. We have shown that there exist three nonsingular hyperelliptic curves $X_{i}$ over $K$ which map onto $E$ via dominant morphisms $\phi_{i}: X_{i} \rightarrow E$ over $K$ for $i=1,2,3$, and this completes the proof.

Remark 5. We note that for an arbitrary elliptic curve $E / K$ and for any integer $g \geq 1$, there exist three hyperelliptic curves $X_{1}, X_{2}, X_{3}$ of genus $1, g, g$ respectively over $\bar{K}$ which are defined as in (*) of Proposition 4. But, in particular, Proposition 4 shows that when $g=3$ we can find three such curves $X_{i}$ defined over $K$ under the assumption that all 2-torsion points of $E / K$ are $K$-rational.

Lemma 6. Let $K$ be an infinite field of finite type and $P_{1}(x), P_{2}(x), \ldots, P_{k}(x)$ a sequence of polynomials in $K[x]$ each of which has a zero of odd multiplicity. If $L / K$ is a finite separable extension of $K$, then there exists $a \in K$ such that $P_{i}(a)$ is not a perfect square in $L$ for $i=1, \ldots, k$.

Proof. See [4, Lemma 4].

Theorem 7. Let $K$ be a number field, $K_{a b}$ the maximal abelian extension of $K$, and $E / K$ an elliptic curve with all $K$-rational 2 -torsion points. Then for each $\sigma \in G_{K}$, the rank of $E\left(\left(K_{a b}\right)^{\sigma}\right)$ is infinite, therefore $E\left(\bar{K}^{\sigma}\right)$ is infinite.

Proof. By Proposition 4 there are three hyperelliptic curves $(*) X_{1}, X_{2}, X_{3}$ defined over $K$ of genus 1,3 , and 3 , respectively forming a biquadratic extension of $\mathbb{P}^{1}$ such that each admits a nonconstant $K$-morphism $\phi_{i}$ onto $E$. 
We inductively construct quadratic extensions $K_{n}$ of $K$ and points $P_{n} \in E\left(K_{n}\right)$ as follows: for each $n$, we apply Lemma 6 to find $x_{n} \in K$ such that

$$
K_{1} K_{2} \cdots K_{n-1}\left(\sqrt{a f\left(x_{n}\right) g\left(x_{n}\right)}, \sqrt{b f\left(x_{n}\right) h\left(x_{n}\right)}\right)
$$

is a biquadratic extension of $K_{1} K_{2} \cdots K_{n-1}$. Then define $L_{n}$ to be the biquadratic extension of $K$ generated by the two square roots $\sqrt{a f\left(x_{n}\right) g\left(x_{n}\right)}, \sqrt{b f\left(x_{n}\right) h\left(x_{n}\right)}$ above. Note that $L_{n}$ contains $\sqrt{a b g\left(x_{n}\right) h\left(x_{n}\right)}$ as well.

Then any element $\sigma \in G_{K}$ induces an action on $L_{n}$ which must fix at least one of the three quadratic $K$-subfields of $L_{n}$. We choose $K_{n}$ to be one of these fixed quadratic subfields of $L_{n}$ over $K$ under $\sigma$. Then one of the three curves $X_{i}$ has a rational point $Q_{n}$ over $K_{n} \subset\left(K_{a b}\right)^{\sigma} \subset \bar{K}^{\sigma}$ with $x$-coordinate $x_{n}$, hence for the curve $X_{i}$ containing $Q_{n}$, the image of $Q_{n}$ under the corresponding $K$-morphism $\phi_{i}$ from $X_{i}$ onto $E$ is a rational point $P_{n}$ of $E\left(K_{n}\right)$ fixed under $\sigma$.

By [5. Lemma], we may assume $P_{n}$ is not a torsion point. Then by the linear disjointness of the quadratic extensions $K_{n}$ over $K$, we can show that the points $P_{n}$ are linearly independent in $E\left(K_{a b}^{\sigma}\right) \otimes \mathbb{Q}$ as in [4, Theorem 5]. Hence, they generate a submodule of $E\left(\prod_{n \geq 1} K_{n}\right) \otimes \mathbb{Q}$ of infinite rank. Since $K_{n}$ are abelian extensions of $K$ and fixed under $\sigma$, we conclude that $E\left(\left(K_{a b}\right)^{\sigma}\right)$ has infinite rank, so $E\left(\bar{K}^{\sigma}\right)$ has infinite rank as well.

Remark 8. For a number field $K$ and an elliptic curve $E / K$, if we let $L$ be a finite extension of $K$ over which all 2-torsion points of $E / K$ are defined, then Theorem 7 implies that for every $\sigma$ in the open subset $\operatorname{Gal}(\bar{K} / L)$ of $G_{K}, E\left(\bar{K}^{\sigma}\right)$ has infinite rank, which implies [4, Theorem 5]. Moreover, since $[L: K] \leq 6$, Theorem 7 gives a bound of 6 on the index of the open subset.

\section{ACKNOWLEDGEMENT}

I wish to thank my thesis advisor, Michael Larsen, for suggesting this problem, his guidance, valuable discussions and helpful comments on this paper. I would like to thank the referee for the careful reading of the original manuscript and many helpful comments and suggestions that improved the presentation. I also thank David Rohrlich for his prompt correspondences and his efficient handling regarding this manuscript.

\section{REFERENCES}

[1] B. Im: Mordell-Weil groups and the rank over large fields of elliptic curves over large fields, arXiv: math.NT/0411533, to appear in Canadian J. Math.

[2] B. Im: Heegner points and Mordell-Weil groups of elliptic curves over large fields, arXiv: math.NT/0411534, submitted for publication, 2003.

[3] S. Lang: Fundamentals of Diophantine Geometry, Springer-Verlag, 1983. MR0715605 (85j:11005)

[4] M. Larsen: Rank of elliptic curves over almost algebraically closed fields, Bull. London Math. Soc. 35 (2003) 817-820. MR2000029 (2004i:11054)

[5] J. H. Silverman: Integer points on curves of genus 1, J. London Math. Soc. (2), 28, (1983) 1-7. MR0703458 (84g:10033)

Department of Mathematics, University of Utah, Salt Lake City, Utah 84112

E-mail address: im@math.utah.edu 